\title{
Process, outcome and experience of transition from child to adult mental healthcare: multiperspective study
}

\author{
Swaran P. Singh, Moli Paul, Tamsin Ford, Tami Kramer, Tim Weaver, Susan McLaren, \\ Kimberly Hovish, Zoebia Islam, Ruth Belling and Sarah White
}

\section{Background}

Many adolescents with mental health problems experience transition of care from child and adolescent mental health services (CAMHS) to adult mental health services (AMHS).

\section{Aims}

As part of the TRACK study we evaluated the process, outcomes and user and carer experience of transition from CAMHS to AMHS.

\section{Method}

We identified a cohort of service users crossing the CAMHS/ AMHS boundary over 1 year across six mental health trusts in England. We tracked their journey to determine predictors of optimal transition and conducted qualitative interviews with a subsample of users, their carers and clinicians on how transition was experienced.

\section{Results}

Of 154 individuals who crossed the transition boundary in 1 year, 90 were actual referrals (i.e. they made a transition to $\mathrm{AMHS}$ ), and 64 were potential referrals (i.e. were either not referred to AMHS or not accepted by AMHS). Individuals with a history of severe mental illness, being on medication or having been admitted were more likely to make a transition than those with neurodevelopmental disorders, emotional/neurotic disorders and emerging personality disorder. Optimal transition, defined as adequate transition planning, good information transfer across teams, joint working between teams and continuity of care following transition, was experienced by less than $5 \%$ of those who made a transition. Following transition, most service users stayed engaged with AMHS and reported improvement in their mental health.

\section{Conclusions}

For the vast majority of service users, transition from CAMHS to AMHS is poorly planned, poorly executed and poorly experienced. The transition process accentuates pre-existing barriers between CAMHS and AMHS

\section{Declaration of interest}

None.
Young people with mental health problems can get 'lost' during transition of care from child and adolescent mental health services (CAMHS) to adult mental health services (AMHS).$^{1-3}$ Disruption of care during transition adversely affects the health, well-being and potential of this vulnerable group. ${ }^{2-9}$ Ideally, transition should be a planned, orderly and purposeful process of change from child-oriented to adult models of care, taking into account both developmental and illness-specific needs. ${ }^{1,10-12}$ If the process is seen simply as an administrative event between CAMHS and AMHS, many health and social care needs may remain unmet. ${ }^{13}$

Transition is often discussed but rarely studied. The national policy in the UK emphasises the need for smooth transition from CAMHS to AMHS ${ }^{2,14-17}$ but there is no published evidence on the process, models and outcomes of transition. A systematic review of 126 papers on transition found only one on a mental health population and only within the US context. ${ }^{5}$ Transition is a critical aspect of continuity of care, yet we know little about who makes such transitions, what are the predictors and outcomes of the process, and how it affects service users and their carers. Without such evidence, mental health services cannot develop and evaluate efficient models that promote successful transition or plan the future development and training programmes to improve transitional care. The TRACK study was designed to answer some of these questions in the UK context.

The overall aims of the TRACK study were to:

1 conduct an audit of the policies and procedures relating to transition within six mental health trusts in London and the West Midlands (three trusts in each region) (Stage 1);
2 evaluate the process of transition by a case-note survey identifying all actual and potential referrals (see below for definitions) from CAMHS to AMHS in the preceding year, 'track' their journey and outcomes in terms of referral and engagement with adult services, and determine the predictors of successful transition (Stage 2);

3 conduct qualitative interviews across organisational boundaries and services within health and social care agencies to identify specific organisational factors that constitute barriers and facilitators to transition and continuity of care (Stage 3);

4 explore the views of service users, carers and mental health professionals on the process of transition experience from a subsample of service users (Stage 4).

In this paper we present findings from Stages 2 and 4. A paper from Stage 1 has already been published. ${ }^{18}$ The TRACK report including Stage 3 findings is available in full at www.sdo.nihr. ac.uk/projdetails.php?ref $=08-1613-117$. The study received ethical approval from Wandsworth Local Research Ethics Committee.

\section{Method}

We used the following definitions: actual referrals were all individuals that crossed the transition boundary and were accepted by AMHS; and potential referrals included individuals that crossed the transition boundary but did not complete transition to AMHS, regardless of the reasons for non-transition. 
Transition pathways were categorised as optimal or sub-optimal. The optimal transition criteria were developed from an audit of CAMHS transition protocols ${ }^{18}$ and literature on good practice in relation to continuity of care. ${ }^{19}$ These criteria included:

(a) information transfer (information continuity): evidence that a referral letter, summary of CAMHS care, or CAMHS case notes were transferred to AMHS along with a contemporaneous risk assessment;

(b) period of parallel care (relational continuity): a period of joint working between CAMHS and AMHS during transition;

(c) transition planning (cross-boundary and team continuity): at least one meeting involving the service user and/or carer and a key professional from both CAMHS and AMHS prior to transfer of care;

(d) continuity of care (long-term continuity) - either engaged with AMHS 3 months post-transition or appropriately discharged by AMHS following transition.

Sub-optimal transitions were those that failed to meet one or more of the above criteria.

\section{Design}

The study was undertaken in six mental health trusts (service provider organisations within the National Health Service), three in Greater London and three in the West Midlands, covering a population of 8.1 million with wide socioeconomic, ethnic and urban-rural heterogeneity. All CAMHS teams that managed young people until the age of transition were included. Highly specialised and tertiary services (e.g. a national eating disorder service) were excluded because of the atypical population served and the logistical problems of tracking individuals from services that accept referrals from across the country.

\section{Case ascertainment}

Case ascertainment was a two-stage process. First, central databases of all included CAMHS were searched for open cases of individuals who had reached age $x$ or above (where $x$ is the age boundary for transition to AMHS). Since the age boundary for different services $(x)$ varied, for each service $x$ was specifically defined as per that service's transition protocol. $^{18}$ In the next stage, all CAMHS clinicians within included services were contacted by letter and email explaining the study and requesting details of actual and potential referrals during the study period. Initial contacts were followed up by further emails and telephone calls during the study period until all clinicians had submitted cases or provided a nil response. For the qualitative study, the young people identified for inclusion were contacted through their care coordinators to explain the study and seek informed consent.

\section{TRACKING tool}

Two data extraction tools, one each for actual and potential referrals were devised and piloted for reliability. Data were collected on sociodemographic and clinical variables, transition pathways and transition outcomes (for actual referrals) and reasons for non-transition in potential referrals. Interrater reliability was checked by two researchers independently extracting data from the tools from five actual referrals from a site unrelated to the project. Comparing 491 non-text variables for each of the five cases, an error rate of less than $2 \%$ was found. Study tools are available in the full study report.

\section{Ascertaining diagnoses}

Since CAMHS case notes vary in recorded diagnoses, we categorised presenting problems into seven diagnostic groups: serious and enduring mental disorders, including schizophrenia, psychotic disorders, bipolar affective disorder, depression with psychosis; emotional/neurotic disorders, including anxiety, depression (without psychosis), post-traumatic stress disorder, obsessive-compulsive disorder; eating disorders, including anorexia nervosa, bulimia nervosa, atypical eating disorder; conduct disorders, including behavioural disorders; neurodevelopmental disorders, including autism-spectrum disorders, intellectual disabilities; substance use disorders, including alcohol and/or drug misuse; and emerging personality disorder. Data on presenting problems were discussed with three CAMHS clinicians (M.P., T.F. and T.K.) to assign a diagnostic group. Comorbidity was defined as the presence of more than one diagnostic category from the seven above.

\section{Predictors of transition}

In the absence of previous evidence, we could not develop a prediction model for transition. Instead a two-stage analysis was conducted with initial identification of independent variables with an association $(P<0.05)$ with the dependent variable using Pearson $\chi^{2}$-tests (Fishers exact tests where necessary) for categorical variables and unpaired $t$-tests for continuous variables. Prior to logistic regression, significant independent variables that were highly associated with each other were recoded into a composite variable to reduce co-linearity. Two logistic regression analyses were planned: first, to determine predictors of achieving transition (being an actual rather than a potential referral); and second, to determine predictors of optimal transition. However, the small numbers of individuals identified in the study who experienced optimal transition precluded the second regression analysis (see Results). It was felt inappropriate to conduct a multilevel analysis as the study did not aim to determine the impact of trust-level variables on transition outcomes. With only six trusts in the sample, there would be insufficient variation in trust-level data for such an analysis. However, to account for possible clustering within trusts, i.e. to account for individuals within trusts being less variable than individuals between trusts, the logistic regression was repeated and standard errors (and therefore 95\% confidence intervals and $P$-values) adjusted for cluster effects (see Results). This analysis was conducted using Stata version 9 for Windows.

\section{Qualitative case studies}

Semi-structured qualitative interviews were conducted with a subsample of service users who had completed transition, and where possible their carers and CAMHS and AMHS care coordinators. A purposive sample of service users $(n=20)$ was initially identified comprising 10 service users, each in two groups: those who did or did not remain engaged with AMHS 3 months post-transition. Within each group we sampled individuals with or without evidence of joint working between CAMHS and AMHS. Within this primary sampling frame we sought to achieve range and diversity in terms of study site, diagnosis, gender, ethnicity and whether or not the service user was an age outlier at time of transition. Service users who declined to participate or who were deemed clinically unsuitable for inclusion were substituted with a matched case. Interviews were conducted by two researchers (K.H. and Z.I.) using topic guides developed by the project team and amended to incorporate emergent themes from all study components. The main focus was on preparation for transition, transition experiences, transition outcomes and 
factors identifiable as related to positive or negative transition outcomes.

\section{Qualitative analysis}

Interviews were recorded, transcribed and entered onto NVivo software (www.qsrinternational.com). K.H. led the development and application of a coding frame with input from Z.I. and the qualitative study lead (T.W.). Use of NVivo facilitated investigator checking of coding. Qualitative analysis was undertaken using the constant comparative method within the framework approach described by Ritchie \& Spencer. ${ }^{20}$ This approach was particularly appropriate for integrating a thematic analysis built upon multiperspective data.

\section{Results}

\section{Quantitative study results}

We encountered major difficulties when searching the central CAMHS databases and these could not be interrogated using the study criteria (see full report for details). We therefore relied primarily on clinician recall to identify cases. A total of 154 individuals were thus identified (London 112; West Midlands 42). The rate of actual and potential referrals per 100000 population in the London sites were 2.68 and 1.49 respectively and in the West Midlands sites 2.23 and 2.97 respectively. The service boundary for transition from CAMHS to AMHS $(x)$ ranged from 16 to 21 years (mode 18).

\section{Transition pathways}

Of the 154 participants, 90 (58\%) were accepted by AMHS (i.e. actual referrals). Sixty-four (42\%) were potential referrals (i.e. those who crossed the transition boundary during the study period but did not make a transition to AMHS). Transition pathways for the entire cohort are shown in Fig. 1.

\section{Sample description}

The total sample consisted of 78 (51\%) males and 76 females with a mean age of 18.12 years (s.d. $=0.82)$. A third $(31 \%)$ were White and $23 \%$ Black and minority ethnic, but ethnicity was not recorded in a large proportion (27\%). The majority (71\%) lived with their parents and nearly two-thirds were either in employment or education (60\%). Diagnostically, half $(n=78)$ had emotional/neurotic disorders, a quarter $(n=38)$ had neurodevelopmental disorders and 22\% $(n=34)$ had serious and enduring mental disorders. Other disorders included substance misuse $(n=14,9 \%)$, conduct disorders $(n=6,4 \%)$, eating disorders $(n=6,4 \%)$ and emerging personality disorder $(n=4$, $3 \%)$. For five individuals $(3 \%)$ the presenting problem was not recorded. Almost a fifth $(n=29,19 \%)$ had comorbid mental health disorders.

\section{Sociodemographic and clinical variables}

Table 1 shows sociodemographic and clinical details of the participants in the actual and potential referrals groups. Those

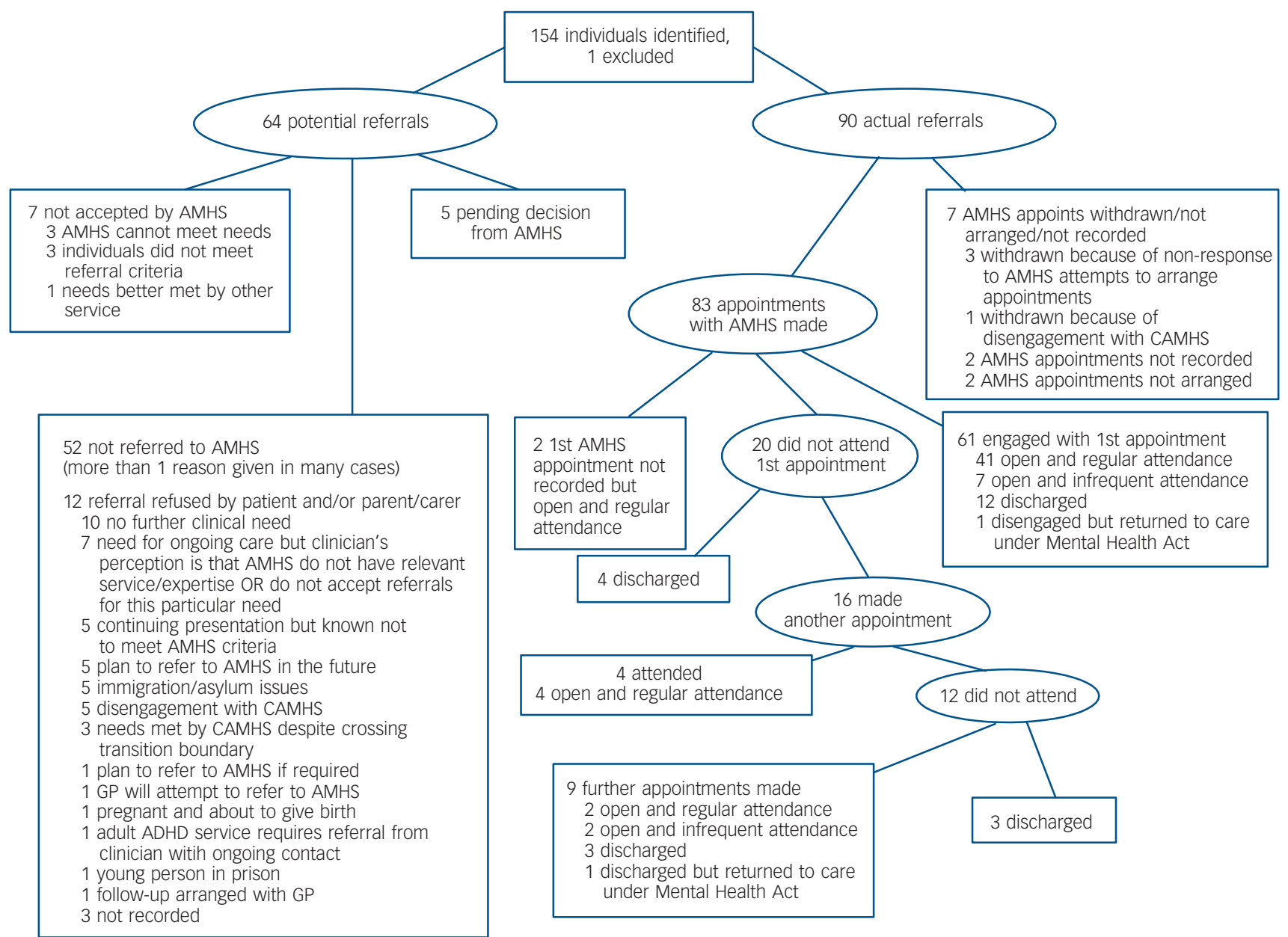

Fig. 1 Outcomes of all referrals from child and adolescent mental health services (CAMHS) to adult mental health services (AMHS). 


\begin{tabular}{|c|c|c|c|c|}
\hline Variable & $\begin{array}{l}\text { Actual referrals group } \\
\qquad(n=90)\end{array}$ & $\begin{array}{l}\text { Potential referrals group } \\
\qquad(n=54)\end{array}$ & $\chi^{2}$ & $P$ \\
\hline Male, $n(\%)$ & $49(54.4)$ & $29(45.3)$ & 1.24 & 0.26 \\
\hline Age at first referral to any CAMHS, years: mean (s.d.) & $13.34(3.9)$ & $14.29(2.9)$ & 1.69 & 0.09 \\
\hline English as first language, $n(\%)$ & $82(91.1)$ & $54(84.4)$ & 1.64 & 0.200 \\
\hline Living with parent, $n$ (\%) & 58 (64.4) & $52(81.3)$ & 6.99 & 0.03 \\
\hline Educational attainment GCSES and below, $n(\%)$ & $43(47.8)$ & $27(42.2)$ & 1.35 & 0.51 \\
\hline Parent attended CAMHS, $n(\%)$ & $34(37.8)$ & $20(31.3)$ & 11.64 & 0.003 \\
\hline Positive family history of mental health problems, $n$ (\%) & $51(56.7)$ & $22(34.4)$ & 3.64 & 0.06 \\
\hline 'Looked after child' while attending CAMHS, $n$ (\%) & $24(26.7)$ & 8 (12.5) & 4.56 & 0.03 \\
\hline Evidence of special educational needs, $n$ (\%) & $19(21.1)$ & $10(15.6)$ & 0.74 & 0.39 \\
\hline Evidence of child protection involvement, $n$ (\%) & $12(13.3)$ & $1(1.6)$ & 6.70 & 0.01 \\
\hline Evidence of youth offending team involvement & $7(7.8)$ & $7(10.9)$ & 0.45 & 0.5 \\
\hline Refugee or asylum seeker status, $n$ (\%) & $10(11.1)$ & $9(14.1)$ & 0.02 & 0.96 \\
\hline Serious and enduring mental illness, $n$ (\%) & $32(35.6)$ & $2(3.1)$ & 22.87 & $<0.0001$ \\
\hline Emotional/neurotic disorder, $n$ (\%) & $43(47.8)$ & $35(54.7)$ & 0.71 & 0.4 \\
\hline Eating disorder, $n(\%)$ & $1(1.1)$ & $5(7.8)$ & 4.49 & 0.03 \\
\hline Conduct disorder, $n$ (\%) & $3(3.3)$ & $3(4.7)$ & 0.81 & 0.67 \\
\hline Substance misuse, $n(\%)$ & $12(13.3)$ & $2(3.1)$ & 4.76 & 0.03 \\
\hline Emerging personality disorder, $n(\%)$ & $4(4.4)$ & 0 & 2.92 & 0.09 \\
\hline Comorbidity (2 or more disorders), $n$ (\%) & $23(25.6)$ & $6(9.4)$ & 6.41 & 0.01 \\
\hline Admitted to hospital while attending CAMHS, $n$ (\%) & $31(34.4)$ & $3(4.7)$ & 19.25 & $<0.0001$ \\
\hline Detained under Mental Health Act, $n(\%)$ & $15(16.7)$ & $1(1.6)$ & 9.16 & 0.002 \\
\hline Risk of self-harm at transition, $n(\%)$ & $5(5.6)$ & $6(9.4)$ & 1.50 & 0.22 \\
\hline On medication at time of transition, $n(\%)$ & $69(76)$ & $29(45)$ & 15.89 & $<0.0001$ \\
\hline
\end{tabular}

in the actual referrals group were more likely to have been living with parents, having attended CAMHS with their parents, to be involved with a child protection agency or be a 'looked after child', been admitted to a psychiatric hospital, to have been detained under the Mental Health Act, to have a serious and enduring mental disorder, substance misuse, an emerging personality disorder or more than one category of presenting problem (comorbidity), but less likely to have an eating disorder. To reduce the number of variables to enter into the logistic regression, a known broader social risk score variable was created that equalled the sum of the following: 'looked after child', child protection involvement, youth offending team involvement, special educational needs or refugee/asylum seeker.

\section{Predictors of transition}

Table 2 shows the results of the logistic regression conducted twice, with the second analysis controlling for clustering within trusts. Having a severe and enduring mental illness and being on medication at the time of transition predicted transition in both analyses. The effect of clustering among trusts was evident in two predictor variables: having 'known broader social risk' and having been admitted for in-patient care.

\section{Optimal transitions}

Based on our four criteria, only 4 of the 90 individuals in the actual referrals group experienced optimal transition. They were 2 males and 2 females, all from Black and minority ethnic backgrounds. Three had a serious and enduring mental disorder and had been admitted to hospital, two under the Mental Health Act. All four were on medication and were from London. Three were referred from an adolescent service.
We were unable to explore predictors of optimal transition given how few individuals had experienced it. We therefore determined predictors of one of the key criterion of optimal transition - continuity of care. This was defined as 'still engaged with AMHS or appropriately discharged 3 months post-transition'. Logistic regression revealed that individuals with emotional/neurotic disorder were a third less likely to experience optimal continuity of care (odds ratio $(\mathrm{OR})=0.34,95 \%$ CI $0.12-0.96, P=0.04$ ). There was no association of continuity of care with any clinical, demographic or process variables.

\section{Qualitative study results}

Of the planned 20 service user participants, we could only interview 11. The most common reason for failing to recruit was no response from the service users to our requests for participation $(25 \%)$. The second most common reason was that a clinician felt that the service user was too ill to participate (18\%). A total of 27 interviews were conducted with 11 service users, 6 parents, 3 CAMHS clinicians and 6 AMHS clinicians.

\section{Emergent themes}

Emergent themes are reviewed briefly below, with some illustrative quotes set out in the online supplement to this paper.

Preparation for transition. Participants described three preparatory mechanisms for transition: transfer planning meetings, joint working and good information transfer. About half (54\%) of young people interviewed reported attending at least one transition planning meeting, usually in the weeks preceding transition, with care coordinators from both CAMHS and AMHS and at least one parent. Service users and carers who did not have 


\begin{tabular}{|c|c|c|c|c|c|}
\hline Independent variable & Odds ratio & $95 \% \mathrm{Cl}$ & $P$ & $95 \% \mathrm{Cl}$, clustered & $P$, clustered \\
\hline Known broader social risk (score) & 1.38 & $0.9-2.1$ & 0.14 & $1.1-1.8$ & 0.02 \\
\hline English as first language & 0.76 & $0.3-2.3$ & 0.62 & $0.4-1.3$ & 0.30 \\
\hline Parents attend CAMHS & 0.56 & $0.2-1.3$ & 0.19 & $0.2-1.3$ & 0.16 \\
\hline Admitted as psychiatric in-patient & 5.05 & $1.0-26.8$ & 0.05 & $0.2-147.3$ & 0.34 \\
\hline Admitted under the Mental Health Act & 5.0 & $0.5-48.3$ & 0.165 & $1.6-15.5$ & 0.01 \\
\hline Eating disorder & 0.24 & $0.0-2.4$ & 0.22 & $0.0-3.4$ & 0.29 \\
\hline Substance misuse & 1.66 & $0.3-11.0$ & 0.59 & $0.3-8.7$ & 0.55 \\
\hline Comorbidity & 2.82 & $0.9-9.4$ & 0.09 & $0.8-9.6$ & 0.01 \\
\hline Serious and enduring illness & 7.85 & $1.6-37.8$ & 0.01 & $1.5-40.9$ & 0.01 \\
\hline On medication at the time of transition & 2.36 & $1.1-5.3$ & 0.04 & $1.7-3.4$ & $<0.01$ \\
\hline
\end{tabular}

transition planning meetings thought that these would have been helpful. Both CAMHS and AMHS clinicians attributed lack of time as a barrier to such meetings. Two service users were told only at their last CAMHS appointment that they were going to be moved to AMHS.

Joint working. Child and adolescent mental health services were generally seen by AMHS colleagues as being in favour of joint working. The AMHS care coordinators appreciated the benefits of joint working (getting to know the service user, being 'in the best interest of the client') but expressed concern about 'responsibility for someone on your case-load, should something go wrong'.

Parental involvement. Parents tended to be less involved with AMHS than with CAMHS. Although young people preferred not having their parents involved in their care any more, parents wanted to be more involved with adult services, in order to be able to express concerns or because they felt 'left in the dark'. One parent stated: 'I know he is now 18 but he is still my son and I worry about him'.

Outcomes of transition. Eight of the eleven young people were still engaged with AMHS at the time of the interview. In most cases $(n=7)$, young people felt that their mental health had improved since the transition to AMHS but did not necessarily attribute this improvement to transition to adult services. Care coordinator flexibility and persistence in the face of missed appointments helped with engagement, although this was more likely to happen when there was evidence of deteriorating mental health or emerging crises. Of the three young people no longer engaged with AMHS, one was discharged as his symptoms had resolved, one did not want to be seen and one was discharged because of non-attendance.

Other transitions. A number of young people experienced other transitions such as change of accommodation or educational status, becoming pregnant or becoming involved with other agencies. Only two young people were still living with their parents after transition. One young person was living with her partner and their child and another was homeless and living in his car. Of the five young women interviewed, three had unplanned pregnancies during the transition period. Four young people had physical health problems closely linked to their mental health and of these, two experienced parallel health service transitions from paediatric to adult care. Five young people had involvement with other services, including Social Services, health visitors, a homeless persons unit, the probation service, school/ education support services, counselling services and an autism support service.

\section{Discussion}

It is a paradox that although treatment for mental disorders in young people have improved substantially in the past two decades, health system responses to young people with mental disorders have been inadequate. ${ }^{21}$ Despite adolescence being a risk period for the emergence of serious mental disorders, substance misuse, other risk-taking behaviours and poor engagement with health services, mental health provision is often patchy during this period. $^{21,22}$ By following a paediatric-adult split, mental health services introduce discontinuities in care provision where the system should be most robust.

To the best of our knowledge, TRACK is the first study in the international literature of the transition process, outcome and experience in a systematically identified cohort of young people who cross the boundary from CAMHS to AMHS. Our biggest methodological challenge was case ascertainment and we were hampered by the poor quality of CAMHS databases. Recall bias among clinicians is likely and our transition rates are certainly underestimates. Additionally, case notes may not accurately reflect the quality and content of services delivered. However, our qualitative results appear to complement the quantitative findings of inadequate transitional care. The requirement of the ethics committee that we seek service user consent through care coordinators meant that we could not interview individuals from the non-referred population (potential referrals) who were invariably out of contact with services. Our catchment was large and diverse, making our findings generalisable to other services in the UK. Internationally, there has been concern about adolescent mental health services in general ${ }^{21,22}$ and about transition in particular. ${ }^{3,23,24}$ Our findings are likely to reflect similar problems internationally.

The findings from TRACK can be summarised as follows: although most service users who crossed the CAMHS transition boundary needed transfer to AMHS, a significant proportion (a third in this study) were not referred to AMHS. Those with neurodevelopmental disorders, emotional/neurotic disorders or emerging personality disorder were most likely to fall through the CAMHS-AMHS gap. Those with a severe and enduring mental illness, a hospital admission and on medication were more likely to make a transition to AMHS but many (a fifth of all actual referrals in this study) were discharged from AMHS care without being seen. Having social risks also predicted transition of care 
when clustering at trust level was taken into account. This might reflect a greater likelihood of the London sample experiencing such risks. Less than $4 \%$ of those accepted by AMHS experienced optimal transition. Although we cannot conclude that optimal transition equates with good clinical outcomes, it certainly equates with good patient experience, a key marker of service quality. In the TRACK cohort, basic principles of good practice identified in transition protocols ${ }^{18}$ were not implemented. For the majority of service users, transition from CAMHS to AMHS was therefore poorly planned, poorly executed and poorly experienced. Transition processes appeared to accentuate all the pre-existing barriers between CAMHS and AMHS. ${ }^{3,25}$

\section{Aligning referral thresholds}

We cannot say why young people with emotional/neurotic, neurodevelopmental or emerging personality disorders are not being referred to AMHS. It is possible that CAMHS may be adjusting their referral thresholds on knowledge and prior experience of local AMHS. If so, this obscures inadequacies in current provision. Where services exist, all young people with ongoing needs should be referred. Where services do not exist, notably those for young people with neurodevelopmental disorders, unmet service user needs should be systematically documented and made clear to AMHS providers and commissioners. Currently, neither CAMHS nor AMHS appear to accept responsibility for the health and welfare of this group. Their outcomes are not known and should be a serious cause for concern.

\section{Transition boundary}

Transition policies in the trusts recommend flexibility regarding transition boundaries based on service user need. ${ }^{18}$ Our study found little evidence of such flexibility. Perhaps services should use 'age windows' to decide the optimal time for transition rather than a strict age cut-off. A crisis should be a relative contraindication to transition; transitions should only be planned and proceed at times of relative stability. There may be situations where transition can only occur during or immediately following a crisis, or where the transition process itself precipitates a crisis, but these occurrences should be relatively rare.

\section{Transition preparation}

Since most transitions can be long anticipated, there should be an adequate period of planning and preparing the service user and their carer for transition. Information about adult services, what to expect, differences in service provision, issues of confidentiality and parental involvement should all form a package of information that CAMHS share with service users and carers prior to transition. The completion of a 'transition logbook' would be a cheap and simple intervention to help structure the transition process. It would be jointly completed by the service user and their care coordinator and contain relevant details such as contact names and numbers, the dates and number of appointments with each agency, the final transition date and service user views on the experience. Such a tool can be easily evaluated on its impact on the process, outcomes and service user experience.

\section{Improving information transfer}

We found that current information technology systems, particularly in CAMHS, did not allow clinicians and managers to access high-quality information on case-loads. Information transfer was also hampered by a lack of understanding of each other's services, inconsistent documentation, different systems used for transfer of electronic information and transfer of referrals to lengthy waiting lists during which professional dialogue was reduced. Inadequate information technology systems in mental health services clearly hinder informational continuity. ${ }^{26}$ The recent National CAMHS Review ${ }^{27}$ notes the frustrations that arise as a result of separate, incompatible information technology systems across different agencies and the need for systems reform and resource support. We recommend that protocols for transition should explicitly specify information that should be transferred between agencies. Where possible, case notes should follow the young person and detailed referral letters, including risk assessments, should be sent to AMHS to facilitate planning. Introduction of electronic records offers an opportunity to facilitate standardisation across services and trusts.

\section{Managing multiple transitions}

Many young people had multiple transitions between AMHS teams, among care coordinators and in their personal circumstances, the cumulative effect of which was complex and unsettling for service users. From our data we cannot tell whether services were unaware of these multiple transitions or unequipped to deal with them. Mental health services, however, must pay attention to these multiple transitions through multi-agency involvement, in order to address the complex needs of this vulnerable group. ${ }^{2,7,28}$

\section{Improving liaison between CAMHS and AMHS}

Maitra \& Jolley ${ }^{29}$ have described a model where child and adult psychiatrists regularly attend each other's clinical meetings at which they jointly address the mental health needs of parents and children within families. Another approach is the development of designated transition workers with posts split between AMHS and CAMHS. ${ }^{4,30,31}$ Such innovations have several benefits, including a higher profile for children and young people within adult services, shaping of the process of referrals across services, improved scope for preventive work, possibilities of joint working and the availability of a forum for formal and informal discussions.

These strategies require closer collaboration between services and agencies, which is demanding of both time and personnel. In periods of fiscal austerity, it is difficult to make a case for enhancing existing services, creating new transition worker posts or developing specialist clinics such as for adults with attentiondeficit hyperactivity disorder. The CAMHS-AMHS divide is also mirrored in the differing commissioning arrangements in the UK, where CAMHS are often commissioned by acute care or children's services, whereas AMHS is firmly within mental health commissioning. Research evidence such as TRACK therefore is the best way for academics and clinicians to influence policy and shape service provision. We believe that joint commissioning between mental health services for children and adults and shared commissioning approaches at a regional level are the best ways to improve transitional care. The Appendix outlines the overall recommendations of the TRACK project. Further recommendations can be found in the full report.

\section{Bridging the divide}

There are two contrasting approaches for improving care for young people undergoing transition from CAMHS to AMHS. We can improve the interface between services as these currently exist, or we can develop a completely new and innovative service 
model of integrated youth mental health services. Each has its advantages, limitations and resource implications. Common to both approaches is the need for services to pay attention to the developmental needs of this age group in areas beyond healthcare transition such as changes in educational and vocational domains, independent living and social and legal status. Although we call for further research into ways of improving transitional care, TRACK findings by themselves demand early and substantial service improvement, some of which can occur without new resources and by simply improving liaison, planning and joint working between CAMHS and AMHS.

In their review of youth mental heath services across the world, Patel et al ${ }^{21}$ concluded: 'our single most important recommendation is the need to integrate youth mental health programmes, including those in the health sector (such as reproductive and sexual health) and outside this sector (such as education)'. The findings of TRACK highlight how far away we are from such integration, given the problems of transition revealed at the interface of CAMHS and AMHS. Even though we do not as yet know how to achieve best transitional care, the status quo of existing service barriers should not be acceptable. We certainly need evidence for any models of transitional care that we test in the future. The search for that evidence should be a goal, rather than a prerequisite for service change. We need to ensure that the vital need for improving youth mental health is not ignored for fear of dismantling long-standing and yet unhelpful service barriers.

Swaran P. Singh, MBBS, MD, FRCPsych, DM, Health Services Research Institute University of Warwick, Coventry; Moli Paul, MRCPsych, PhD, University of Warwick, Coventry; Tamsin Ford, MRCPsych, PhD, Peninsula College of Medicine and Dentistry, Plymouth; Tami Kramer, MRCPsych, Tim Weaver, PhD, Imperial College London, London; Susan McLaren, BSC, PhD, London Southbank University, London; Kimberly Hovish, BA, MSc, Institute of Education, London; Zoebia Islam, MSc, PhD, Birmingham \& Solihull Mental Health Foundation Trust, Birmingham; Ruth Belling, BLib, Joint Hons, PhD, London Southbank University, London; Sarah White, MSC, St George's University of London, London, UK

Correspondence: Swaran P. Singh, Health Services Research Institute, Medica School Building, Gibbet Hill Campus, University of Warwick, Coventry CV4 7AL, UK. Email: S.P.Singh@warwick.ac.uk

First received 4 Nov 2009, final revision 21 Jun 2010, accepted 24 Jun 2010

\section{Funding}

The TRACK study is funded by the National Institute of Health Research (NIHR) Service Delivery and Organisation (SDO) programme (www.sdo.nihr.ac.uk). The views and opinions expressed therein are those of the authors and do not necessarily reflect those of the NIHR SDO programme or the Department of Health.

\section{Acknowledgements}

The TRACK study team included Professor Swaran Singh, Dr Ruth Belling, Dr Jenny Dale, Dr Navina Evans, Dr Tamsin Ford, Dr Nicole Fung, Ms Katherine Harley, Dr Daniel Hayes, Ms Kimberly Hovish, Dr Zoebia Islam, Dr Bob Jezzard, Dr Tami Kramer, Professor Susan McLaren, Dr Moli Paul, Dr Anne Rourke, Dr Tim Weaver and Dr Sarah White.

\section{Appendix}

\section{Overall recommendations from the TRACK study}

(a) The needs of the service user should be central to protocol and service development regarding transition.

(b) Trusts should have regular updated mapping of local CAMHS, AMHS and voluntary services, identifying scope of operation, communication networks and key contacts.

(c) Protocols should be developed and implemented in collaboration with all relevant agencies and young people and their carers. (d) Multidisciplinary training should be planned and delivered for transition, including local service structures, protocols and working with young people. This training should be linked to the appraisal process and skills and competency frameworks.

(e) Protocols should specify the time frame, lines of responsibility and who should be involved, how the young person should be prepared and what should happen if AMHS are unable to accept the referral.

( $)$ Protocols should stress flexibility in the age range to accommodate a range of needs and developmental stages, and have explicit referral criteria and service provision.

(g) Transition should occur at times of stability where possible; young people should not have to relapse in order to access a service.

(h) Agencies should try to avoid multiple simultaneous transitions

(i) Improved information transfer between CAMHS/AMHS with the standardisation of record keeping or, where this is impossible, clear indication of what information should be made available. A referral letter summarising past contact, current state and risks is a bare minimum. If all records cannot be transferred, copies of all correspondence and contact summaries should be.

(j) Transition process should include collaborative working between CAMHS and AMHS, with cross-agency working or periods of parallel care.

(k) Carers' needs and wishes should be respected in the transition process and carer involvement in adult services should be sensitively negotiated between clinicians, service users and their carers.

(I) Services need to develop for young people with emotional/neurotic, emerging personality and neurodevelopmental disorders wherever there is gap in such provision.

(m) Active involvement by AMHS is required before CAMHS can discharge a case; transfer onto a long waiting list is unacceptable.

(n) Changes should be evidence based. Prospective research is required on the clinical course, service needs, health and social cost implications for the young people receiving little service provision after leaving CAMHS.

\section{References}

1 Royal College of Paediatrics and Child Health. Bridging the Gaps: Health Care for Adolescents. Royal College of Paediatrics and Child Health, 2003 (http:// www.rcpch.ac.uk/).

2 Lamb C, Hill D, Kelvin R, Van Beinum M. Working at the CAMHS/Adult Interface: Good Practice Guidance for the Provision of Psychiatric Services to Adolescents/Young Adults. A Joint Paper from the Interfaculty Working Group of the Child and Adolescent Faculty and the General and Community Faculty of the Royal College of Psychiatrists, May 2008. Royal College of Psychiatrists, 2008.

3 Singh SP. Transition of care from child to adult mental health services: the great divide. Curr Opin Psychiatry 2009; 22: 386-90.

4 Forbes A, While A, Ullman R, Lewis S, Mathes L, Griffiths $\mathrm{P}$, et al. A MultiMethod Review to Identify Components of Practice which may Promote Continuity in the Transition from Child to Adult Care for Young People with Chronic Illness or Disability. Report for the National Coordinating Centre for NHS Service Delivery and Organisation R \& D. NCCSDO, 2002 (http:// www.sdo.Ishtm.ac.uk)

5 While A, Forbes A, Ullman R, Lewis S, Mathes L, Griffiths P. Good practices that address continuity during transition from child to adult care: synthesis of the evidence. Child Care Health Dev 2004; 30: 439-52.

6 Kennedy A, Douglass S. Young people with chronic illness: the approach to transition. Intern Med J 2007; 37: 555-60.

7 Department for Children Schools and Families and Department of Health Transition: Moving on Well. A Good Practice Guide for Health Professionals and their Partners on Transition Planning for Young People with Complex Health Needs or a Disability. Department of Health, 2008.

8 Department of Health. Good Transitions for Good People. Department of Health, 2006.

9 American Academy of Pediatrics, American Academy of Family Physicians American College of Physicians-American Society of Internal Medicine. A consensus statement on health care transitions for young adults with special health care needs. Pediatrics 2002; 110: 1304-6. 
10 Blum RW, Garell D, Hodgman CH, Jorissen TW, Okinow NA, Orr DP, et al. Transition from child-centered to adult health-care systems for adolescents with chronic conditions: a position paper for the Society of Adolescent Medicine. J Adolesc Health 1993; 14: 570-6.

11 McDonagh JE, Kelly DA. Transiting care of the pediatric recipient to adult care givers. Pediatr Clin North Am 2003; 50: 1561-83.

12 Royal College of Nursing. Adolescent Transition Care. RCN Publications, 2004

13 Vostanis P. Patients as parents and young people approaching adulthood: how should we manage the interface between mental health services for young people and adults? Curr Opin Psychiatry 2005; 18: 449-54.

14 Commission for Health Improvement. Transition of Care between CAMHS and Adult Services. Commission for Health Improvement, 2003 (http:// www.chi.nhs.uk/Ratings/Trust/Indicator/indicatorDescriptionshort. asp? indicatorld=3555).

15 Department of Health. Getting the Right Start: National Service Framework for Children: Emerging Findings. Department of Health, 2003.

16 Department for Education and Skills. Every Child Matters: Change for Children. Department for Education and Skills, 2004.

17 Department of Health and Department for Education and Skills. National Service Framework for Children, Young People and Maternity Services. Department of Health, 2004.

18 Singh SP, Paul M, Ford T, Kramer T, Weaver T. Transitions of care from child and adolescent mental health services to adult mental health services (TRACK study): a study of protocols in Greater London. BMC Health Serv Res 2008; 8: 1-7.

19 Freeman G, Crawford M, Weaver T, Low J, de Jonge F. Promoting Continuity of Care for People with Severe Mental IIIness whose needs span Primary, Secondary and Social Care: A Multi-Method Investigation of Relevant Mechanisms and Contexts. NCCSDO, 2002.

20 Ritchie J, Spencer L. Qualitative data analysis for applied policy research. In Analysing Qualitative Data (eds A Bryman, R Burgess): 172-94. Routledge 1994
21 Patel V, Flisher AJ, Hetrick S, McGorry P. Mental health of young people: a global public-health challenge. Lancet 2007; 369: 1302-13.

22 McGorry P. The specialist youth mental health model: strengthening the weakest link in the public mental health system. Med J Aust (suppl) 2007; 187: s53-6.

23 Cosgrave EM, Yung AR, Killackey E, Buckby JA, Godfrey, KA, Standford CA, et al. Met and unmet need in youth mental health. J Ment Health 2008; 17 618-28

24 Davis M, Geller JL, Hunt B. Within-state availability of transition-to-adulthood services for youths with serious mental health conditions. Psychiatr Serv 2006; 57: 1594-9.

25 Singh SP, Evans N, Sireling, L, Stuart H. Mind the gap: the interface between child and adult mental health services. Psychiatr Bull 2005 29: 292-4.

26 Burns T, Clement S, Cathy J, McLaren S, Rees-Jones, I, Wykes T, et al. Experiences of Continuity of Care and Health and Social Outcomes: The ECHO Study. National Coordinating Centre for Service Delivery and Organisation, 2007.

27 National CAMHS Review. Children and Young People in Mind: the Final Report of the National CAMHS Review. Department for Children, Schools and Families and Department of Health, 2008.

28 Department for Education and Skills and Department of Health. Report on the Implementation of Standard 9 of the National Service Framework for Children, Young People and Maternity Services. Department of Health, 2006.

29 Maitra B, Jolley A. Liaison between child and adult psychiatric services. In Family Matters: Interface Between Child and Adult Mental Health (eds P Reder, M McClure, A Jolley): 285-302. Routledge, 2000.

30 Health and Social Care Advisory Service. CAMHS to Adult Transition: $A$ Literature Review for Informed Practice. Health and Social Care Advisory Service (HASCAS) Tools for Transition. Department of Health and HASCAS 2006 (http://www.hascas.org.uk/pdf files/HASCAS\%20tools\%20for\%20 transition\%20Lit\%20Review.pdf).

31 Social Exclusion Unit. Transitions: Young Adults withComplex Needs. Office of the Deputy Prime Minister, 2005.

\title{
Edvard Munch (1863-1944)
}

\author{
Alexandra Pitman
}

The Norwegian Expressionist Edvard Munch caused outrage when his paintings were first shown in Berlin but became one of the most prolific artists of his time. Often described as having had bipolar affective disorder, his low moods and sense of isolation are evident in works such as The Scream, Separation, and Evening on Karl Johan. Yet the evidence of his diaries and his many biographies suggest more plausible diagnoses of depressive disorder and comorbid alcohol dependence. Art historians acknowledge his ability to represent extreme emotional states, while debating the extent to which Munch exploited the market for his 'flawed personality'. 